\title{
Tocilizumab may slow radiographic progression in patients with systemic or polyarticular-course juvenile idiopathic arthritis: post hoc radiographic analysis from two randomized controlled trials
}

Clara Malattia ${ }^{1,2}$, Nicolino Ruperto², Silvia Pederzoli², Elena Palmisani ${ }^{2}$, Angela Pistorio ${ }^{3}$, Carine Wouters ${ }^{4}$, Pavla Dolezalova ${ }^{5}$, Berit Flato ${ }^{6}$, Stella Garay ${ }^{7}$, Gabriella Giancane ${ }^{2}$, Chris Wells ${ }^{8}$, Wendy Douglass ${ }^{8}$,

Hermine I. Brunner ${ }^{9}$, Fabrizio De Benedetti ${ }^{10}$, Angelo Ravelli ${ }^{1,2,11^{*}}$ (D) and for the Paediatric Rheumatology International Trials Organisation (PRINTO) and the Pediatric Rheumatology Collaborative Study Group (PRCSG)

\begin{abstract}
Background: Few clinical trials have investigated the prevention of radiographic progression in children with juvenile idiopathic arthritis treated with antirheumatic drugs. This study aimed to investigate radiographic progression in patients with systemic juvenile idiopathic arthritis (s/A) and patients with polyarticular-course juvenile idiopathic arthritis (pcJIA) treated with the anti-interleukin-6 receptor antibody tocilizumab for 2 years in the TENDER and CHERISH randomized controlled trials, respectively.

Methods: Standard radiographs of both wrists and both hands in the posteroanterior view were obtained within 4 weeks of baseline and were repeated at weeks $52 \pm 4$ and $104 \pm 4$ in both trials. All films were scored by two independent readers using the adapted Sharp-van der Heijde (aSH) and Poznanski scoring methods. Although the Poznanski score indicates bone growth limitation or cartilage growth decrease, which are not the same as joint space narrowing in rheumatoid arthritis, its change reflects damage to cartilage. Therefore, impairment in the Poznanski score as well as the aSH score was considered as a measure of structural joint damage. Radiographic progression was defined as worsening of radiographic scores beyond the smallest detectable difference.

(Continued on next page)
\end{abstract}

\footnotetext{
* Correspondence: angeloravelli@gaslini.org

'Università degli Studi di Genova, Genoa, Italy

${ }^{2}$ IRCCS Istituto Giannina Gaslini, Clinica Pediatrica e Reumatologia, PRINTO,

Via G. Gaslini 5, 16147 Genoa, Italy

Full list of author information is available at the end of the article
}

(c) The Author(s). 2020 Open Access This article is licensed under a Creative Commons Attribution 4.0 International License, which permits use, sharing, adaptation, distribution and reproduction in any medium or format, as long as you give appropriate credit to the original author(s) and the source, provide a link to the Creative Commons licence, and indicate if changes were made. The images or other third party material in this article are included in the article's Creative Commons licence, unless indicated otherwise in a credit line to the material. If material is not included in the article's Creative Commons licence and your intended use is not permitted by statutory regulation or exceeds the permitted use, you will need to obtain permission directly from the copyright holder. To view a copy of this licence, visit http://creativecommons.org/licenses/by/4.0/. The Creative Commons Public Domain Dedication waiver (http://creativecommons.org/publicdomain/zero/1.0/) applies to the data made available in this article, unless otherwise stated in a credit line to the data. 
(Continued from previous page)

Results: Poznanski and aSH scores were available at baseline and at one or more postbaseline time points for 33 and 47 of $112 \mathrm{~s} J \mathrm{~A}$ patients and 61 and 87 of 188 pcJIA patients, respectively, providing a representative subset of the study populations. The inter-reader and intra-reader agreement intra-class correlation coefficient was $>0.8$. Median baseline Poznanski and aSH scores, respectively, were -2.4 and 24.6 for sJIA patients and -1.5 and 8.0 for pcJIA patients. Compared with baseline, aSH scores remained stable for all sJA patients at week 52 , whereas $9.4 \%$ of SJIA patients had radiographic progression according to Poznanski scores at week 52; at 104 weeks, radiographic progression according to aSH and Poznanski scores was observed in 5.4\% and 11.5\%, respectively. In pcJIA patients, radiographic progression from baseline at 52 weeks and at 104 weeks was $12.5 \%$ and 2.9\%, respectively, using aSH scoring and $6.5 \%$ and $4 \%$, respectively, using Poznanski scoring.

Conclusion: Tocilizumab may delay radiographic progression in children with SJIA and children with pcJIA.

Trial registration: Trial registration numbers and dates: TENDER, NCT00642460 (March 19, 2008); CHERISH, NCT00988221 (October 1, 2009)

Keywords: Biologicals, Disease-modifying antirheumatic drugs (DMARDs), Tocilizumab, Systemic juvenile idiopathic arthritis, Polyarticular-course juvenile idiopathic arthritis

\section{Background}

Juvenile idiopathic arthritis (JIA) encompasses a heterogeneous group of immune-mediated, chronic, noninfectious joint diseases commencing before patients are 16 years of age [1]. All JIA types are characterized by chronic synovial inflammation, potentially leading to permanent damage of articular cartilage and bone [2]. Joint space narrowing, reflecting cartilage loss over the joint surface, can result in serious impairment of physical function [3]. Many children with JIA develop marked radiographic joint damage, with cartilage loss and erosions often developing early in the course of their illness [4]. Despite the recent development and validation of scoring methods [4-12], the capacity of antirheumatic medications to prevent radiographic progression has not been extensively investigated [13-16].

Tocilizumab is a humanized anti-human interleukin-6 (IL-6) receptor-alpha antibody that inhibits IL-6 signaling $[17,18]$. Based on results from phase 3 randomized controlled trials in patients with systemic JIA (sJIA [TENDER trial]) and polyarticular-course JIA (pcJIA [CHERISH trial]) [19, 20], intravenous tocilizumab, in combination with methotrexate or as monotherapy, was approved for the treatment of both subtypes of JIA [21]. Tocilizumab has been shown to significantly slow radiographic joint damage in adults with rheumatoid arthritis [22-24] and to reduce radiographic abnormalities in a small sample of patients with sJIA [25].

The present analysis was conducted to investigate the effect of tocilizumab on radiographic progression for up to 2 years in patients with sJIA or pcJIA from the TEND ER and CHERISH phase 3 randomized controlled trials.

\section{Patients and methods}

\section{Patients}

Eligibility criteria have been published [19, 20]. Briefly, eligible patients were 2 to 17 years of age, had a diagnosis of
sJIA or pcJIA (rheumatoid factor-positive polyarthritis, rheumatoid factor-negative polyarthritis, or extended oligoarthritis) according to the International League of Associations for Rheumatology classification criteria [1], and had active disease for $\geq 6$ months. At enrollment, patients with sJIA had to have $\geq 5$ active joints or fever $>38^{\circ} \mathrm{C}$ and $\geq 2$ active joints and inadequate response to nonsteroidal anti-inflammatory drugs and glucocorticoids. Patients with pcJIA had to have $\geq 5$ active joints and inadequate response or intolerance to methotrexate. Stable doses of methotrexate, nonsteroidal anti-inflammatory drugs, and/ or oral glucocorticoids (maximum dose $0.5 \mathrm{mg} / \mathrm{kg} /$ day for sJIA, $0.2 \mathrm{mg} / \mathrm{kg} /$ day for pcJIA) were allowed throughout the studies. Children with wrist involvement were included if consent for X-ray was provided.

\section{Study design}

The sJIA trial was conducted across 43 centers in 17 countries, and the pcJIA trial was conducted at 58 centers in 15 countries. All centers are part of the Paediatric Rheumatology International Trials Organisation (PRIN TO) [26] or the Pediatric Rheumatology Collaborative Study Group (PRCSG). Study designs have been described $[19,20]$. Briefly, the sJIA trial was a 5-year, phase 3 study of the efficacy and safety of tocilizumab in patients with active sJIA, with a 12-week, randomized, double-blind, placebo-controlled period followed by a long-term extension. The pcJIA trial was a 2-year, phase 3 randomized withdrawal trial of the efficacy and safety of tocilizumab in patients with pcJIA, with a 16-week open-label, lead-in period; a 24-week double-blind, placebo-controlled withdrawal period; and a long-term extension. Both trials were conducted in accordance with the Declaration of Helsinki and good clinical practice and were approved by the local institutional review 
board or the independent ethics committees at each center.

\section{Radiographic assessments}

Standard radiographs of both wrists and both hands in the posteroanterior view were obtained for a subset of patients who consented to X-ray within 4 weeks of baseline and again at week 52 ( \pm 4 weeks) and at week 104 ( \pm 4 weeks). All radiographs were scored independently by two pediatric rheumatologists $(\mathrm{CM}, \mathrm{AR})$ with $>10$ and $>20$ years of experience, respectively, in clinical and radiographic assessment of children with JIA using the adapted Sharp-van der Heijde (aSH) [11] and Poznanski [27] scoring methods. Radiographs from each patient were read in random order; previous radiographs and scores were not available to readers when they were examining and scoring follow-up radiographs. Inter-reader reliability methods are described in the Additional File.

Calculation of aSH scores was based on assessment of 15 areas for joint space narrowing (JSN) and 21 areas for erosion in each hand and wrist [11]. For each area, JSN was scored from 0 to $4(0=$ normal, $1=$ focal or minimal narrowing, $2=$ loss of joint space $<50 \%, 3=$ loss of joint space $>50 \%, 4=$ ankylosis $)$ and erosion from 0 to $5(0=$ normal shape, 1 = slight deformity, $2=$ moderate deformity, $3=$ marked deformity, $4=$ severe deformity $5=$ extensive bone destruction). aSH scores from each of the two independent readers were averaged for each of the areas assessed. Scores were calculated from the unweighted summary score of 15 area JSN (range, 0-120) and 21 area erosion (range, 0-210), with the total aSH score (range, 0-330) representing the sum of the JSN and erosion scores and higher scores indicating greater damage. Further details are described in the Additional File.

The Poznanski method is based on measurements of the radial metacarpal (RM) width, which is the distance from the base of the third metacarpal bone to the midpoint of the distal growth plate of the radius, and the maximum length of the second metacarpal bone (M2). Although the Poznanski score indicates bone growth limitation or cartilage growth decrease, which are not the same as joint space narrowing in rheumatoid arthritis, its change reflects damage to cartilage. Therefore, impairment in the Poznanski score as well as the aSH score was considered as a measure of structural joint damage. For each wrist, the number of standard deviations between the expected and the observed RM width for the measured M2 length was calculated according to the formulae of Poznanski et al. [27]. The RM/M2 ratio, which represents the Poznanski score, reflects the amount of radiographic damage in the wrist. The more negative the Poznanski score, the more severe the radiographic damage. Further details are described in the Additional File.
Importantly, the radiographic scores obtained with the two methods used in our study go in opposite directions. With the Poznanski score, a decrease is abnormal, whereas with the aSH score an increase is abnormal.

Key radiographic end points were change from baseline in radiographic scores, proportion of patients without radiographic progression from baseline to weeks 52 or 104, and relationship between radiographic progression and clinical response.

Radiographic progression at 1 and 2 years was determined by subtracting baseline scores from week 52 and 104 scores. Radiographic progression for aSH and Poznanski scores was defined using the smallest detectable difference (SDD) and the zero value (Additional File) [28]. Positive change in aSH score [11] or negative change in Poznanski score [27], or both, was considered indicative of radiographic progression. Data are reported using Bland and Altman plots. Further details are described in the Additional File.

Change from baseline to week 104 in aSH score was analyzed for patients in the pcJIA radiographic population by stratifying patients according to baseline methotrexate use (yes/no), baseline glucocorticoid use (yes/ no), previous biologic use (yes/no), disease duration $(<2$ years $/ \geq 2$ years), and rheumatoid factor (positive/negative/missing).

\section{Statistical analysis}

Radiographic end points were evaluated for the entire radiographic population of the sJIA trial, whereas analysis of the pcJIA trial focused on the subgroup of radiographic patients who received tocilizumab continuously throughout the study (those randomly assigned to receive tocilizumab during the double-blind period [continuous TCZ population]). Some radiographic analyses were performed for the entire radiographic population (all patients with radiographic data regardless of randomly assigned placebo or tocilizumab treatment [all TCZ population]). The aSH population included all patients who received a dose of tocilizumab and had a baseline and $\geq 1$ postbaseline (week 52 or week 104) score, with data at weeks 52 and 104 summarized for patients who received tocilizumab continuously. The Poznanski population included all patients who received $\geq 1$ dose of tocilizumab and had $\geq 1$ postbaseline (week 52 or week 104) score, with data at weeks 52 and 104 summarized for patients who received tocilizumab continuously.

Missing readings of areas for JSN or erosion in the $\mathrm{aSH}$ score, because of either absence of a particular area due to incomplete ossification (typically seen in younger children) or technical inadequacy of the X-ray film, were imputed using average readings from other readable areas. If readings from an entire hand or wrist were missing, erosion, JSN, and total adapted aSH scores were 
set to missing. No imputation was used for missing Poznanski score data or other analyses.

Changes from baseline in aSH and Poznanski scores were analyzed using the Wilcoxon signed rank test. Correlation between aSH and Posnanski scores was assessed using Pearson and Spearman correlations. Annualized rates of progression were calculated as the change from baseline of each follow-up visit, divided by the number of days between the two assessments, and multiplied by 365.25 .

\section{Results}

\section{Radiographic populations and baseline characteristics}

In total, 112 patients were enrolled in the sJIA trial and received $\geq 1$ dose of tocilizumab. Baseline and $\geq 1$ postbaseline aSH scores and Poznanski scores were assessed for 47 and 33 patients, respectively, from 25 of 43 (58\%) centers in 14 of $17(82 \%)$ countries. The aSH population included 45 and 37 patients at weeks 52 and 104, respectively, and the Poznanski population included 32 and 26 patients at weeks 52 and 104, respectively.

In total, 188 patients were enrolled in the pcJIA trial and received $\geq 1$ dose of tocilizumab. Baseline and $\geq 1$ postbaseline aSH scores and Poznanski scores were assessed for 87 and 61 patients, respectively, from 25 of
$43(58 \%)$ centers in 14 of $17(82 \%)$ countries. In the pcJIA study, 45 patients in the aSH population and 35 patients in the Poznanski population received tocilizumab continuously (continuous TCZ population). The aSH population included 40 and 35 patients at weeks 52 and 104, respectively, and the Poznanski population included 31 and 25 patients at weeks 52 and 104, respectively.

For both studies, baseline demographics and disease characteristics of patients who underwent radiographic assessments were similar to those of the total populations $[19,20]$ (Table 1).

Notably, the Poznanski score could not be assessed in patients with advanced carpometacarpal erosions that made it difficult to define the bone ends or in older children who had apparent radiographic closure of the second metacarpal growth plate. These phenomena did not preclude assessment of the aSH score, which explains why patients with available aSH scores outnumber those who had the Poznanski score calculated.

\section{Inter-reader and intra-reader reliability}

Inter-reader and intra-reader agreement, as assessed by intra-class correlation coefficient (ICC) for radiographic

Table 1 Baseline demographics and disease characteristics of patients with sJIA and pcJIA (radiographic and study populations)

\begin{tabular}{|c|c|c|c|c|c|c|}
\hline \multirow[t]{2}{*}{ Characteristic } & \multicolumn{3}{|l|}{ sJIA } & \multicolumn{3}{|l|}{$\mathrm{pcJ} / \mathrm{A}$} \\
\hline & Adapted SH, $n=47$ & Poznanski, $n=33$ & All, $n=112$ & Adapted SH, $n=45$ & Poznanski, $n=35$ & All, $n=188$ \\
\hline Age, years & $9.9(4.3)$ & $8.4(4.2)$ & $9.7(4.6)$ & $10.8(3.7)$ & $9.9(3.3)$ & $11.0(4.0)$ \\
\hline Female, $n(\%)$ & $24(51)$ & $13(39)$ & $56(50)$ & $34(76)$ & $27(77)$ & $144(77)$ \\
\hline Race, white, $n(\%)$ & $43(91)$ & $29(88)$ & $99(88)$ & $33(73)$ & $25(71)$ & $150(80)$ \\
\hline Body weight, kg & $33.7(15.7)$ & $28.2(14.5)$ & $33.8(19.6)$ & $39.3(16.3)$ & $36.3(14.7)$ & $39.6(17.3)$ \\
\hline Disease duration, years & $5.2(4.2)$ & $4.8(4.1)$ & $5.2(4.1)$ & $3.9(3.3)$ & $3.2(2.4)$ & $4.2(3.7)$ \\
\hline Joints with active arthritis ${ }^{a}$ & $21.3(15.7)$ & $19.2(16.5)$ & $19.8(15.7)$ & $20.9(13.7)$ & $21.7(14.5)$ & $20.3(14.3)$ \\
\hline Joints with LOM ${ }^{b}$ & $20.0(15.7)$ & $18.2(16.7)$ & $19.8(15.6)$ & $14.8(12.0)$ & $16.3(13.0)$ & $17.6(14.4)$ \\
\hline Patient/parent global assessment VAS ${ }^{c}$ & $55.4(22.7)$ & $55.6(25.1)$ & $58.7(24.4)$ & $42.5(26.3)$ & $41.9(26.4)$ & $52.9(25.0)$ \\
\hline Physician global assessment VAS ${ }^{c}$ & $62.3(19.5)$ & $62.8(20.0)$ & $64.9(22.3)$ & $57.2(19.8)$ & $59.1(18.4)$ & $61.4(20.7)$ \\
\hline CHAQ-DI score (0-3) & $1.6(0.9)$ & $1.6(0.9)$ & $1.7(0.9)$ & $1.3(0.6)$ & $1.3(0.5)$ & $1.4(0.7)$ \\
\hline $\mathrm{ESR}, \mathrm{mm} / \mathrm{h}$ & $53.9(31.5)$ & $59.2(35.2)$ & $57.6(34.2)$ & $29.9(22.3)$ & $30.9(21.9)$ & $34.8(25.5)$ \\
\hline Previous DMARDs use, $n(\%)$ & $34(72)$ & $22(67)$ & $82(73)$ & $31(69)$ & $20(57)$ & $134(71)$ \\
\hline Previous biologic use, $n(\%)$ & $39(83)$ & $26(79)$ & $92(82)$ & $9(20)$ & $7(20)$ & $61(32)$ \\
\hline Background methotrexate use, $n(\%)$ & $34(72)$ & $28(85)$ & $77(69)$ & $39(87)$ & $30(86)$ & $148(79)$ \\
\hline Methotrexate dose, $\mathrm{mg} / \mathrm{m}^{2} /$ week & - & - & - & $12.5(3.3)$ & $12.7(3.3)$ & $13.0(5.8)$ \\
\hline Background oral GC use, $n$ (\%) & $23(49)$ & $12(36)$ & $55(49)$ & $19(42)$ & $15(43)$ & $86(46)$ \\
\hline GC dose, $\mathrm{mg} / \mathrm{kg} / \mathrm{day}^{\mathrm{d}}$ & $0.28(0.17)$ & $0.31(0.16)$ & $0.30(0.20)$ & $0.13(0.05)$ & $0.14(0.05)$ & $0.13(0.05)$ \\
\hline
\end{tabular}

Data are mean (SD) unless otherwise noted

Abbreviations: CHAQ-DI Childhood Health Assessment Questionnaire-Disability Index, DMARDs disease-modifying antirheumatic drugs, ESR erythrocyte

sedimentation rate, GC glucocorticoid, LOM limitation of motion, $p C J I A$ polyarticular-course juvenile idiopathic arthritis, SD standard deviation, SH Sharp-van der

Heijde, sJIA systemic juvenile idiopathic arthritis, VAS visual analog scale

a 71 -joint count

b67-joint count

${ }^{\mathrm{c}} 0-100 \mathrm{~mm}$

dPrednisone equivalent 
scores, was good for both JIA subtypes and for both scoring methods, with all ICCs $>0.8$ and most $>0.9$ (results not shown). Agreement between readers was confirmed by Bland and Altman plots (Figs. 1 and 2).

\section{Radiographic progression in patients with sالA}

Median [interquartile range] changes from baseline in total aSH scores were not significant at weeks $52(0.00$ $[-8.70,4.00]) ; P=0.302)$ and $104(0.50[-7.50,12.00]$; $P=0.695)$ (Fig. 3a, Table 2). Similarly, no significant change from baseline was observed in erosion scores at weeks $52(0.50[-3.50,1.50] ; P=0.677)$ and $104(0.50[-$ $1.00,4.50] ; P=0.257)$ or in JSN scores at weeks $52(0.00$ $[-4.00,3.00] ; P=0.257)$ and $104(0.00[-5.50,4.00] ; P=$ 0.937) (Table 2). Median [interquartile range] Poznanski scores increased significantly from baseline to week 52 (0.29 $[-0.05,1.05] ; P=0.003)$, but change from baseline to week 104 was not significant $(0.16[-0.01,1.04] ; P=$ 0.057 ) (Fig. 3b, Table 2). There was a weak negative correlation between aSH and Poznanski scores at week 52 (Pearson correlation, - 0.233; Spearman correlation, 0.121 ) and a weak-to-moderate negative correlation at week 104 (Pearson correlation, - 0.682; Spearman correlation, - 0.303).
The SDD for aSH score progression was 23.6 at week 52 and 27.4 at week 104 . Using the SDD of $23.6,100 \%$ and $94.6 \%$ of tocilizumab-treated patients showed no aSH progression at weeks 52 and 104, respectively (Fig. 1a, b). Proportions of patients without progression-using a cutoff of zero-are described in the Additional File.

The SDD for Poznanski score progression was 0.58 at week 52 and 0.74 at week 104. Based on the SDD of 0.58, 90.6\% and $88.5 \%$ of patients did not experience Poznanski progression at weeks 52 and 104, respectively (Fig. 1c, d).

Mean annualized progression rates from baseline to week 104 were $0.29,0.44$, and -0.15 for total, erosion, and JSN aSH scores, respectively, and -0.18 for Poznanski score, indicating a lack of radiographic progression over 2 years.

The clinical effectiveness of tocilizumab, assessed by JIA American College of Rheumatology (ACR) response criteria [29], did not appear to be related to radiographic progression. All patients with available radiographs achieved JIA ACR50 response or higher, except for one who was a nonresponder at week 52 but who achieved JIA ACR70 response by week 104; this patient did not experience aSH or Poznanski progression according to SDD. Two patients who exhibited aSH progression at week 104 (based on SDD calculation) met JIA ACR90

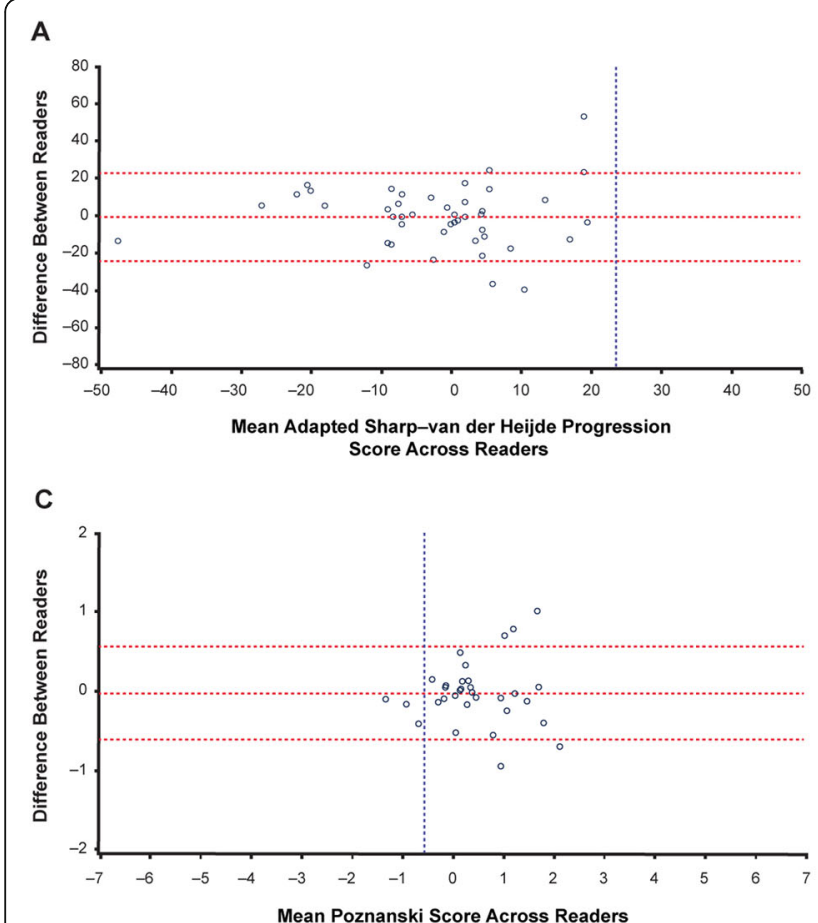

B

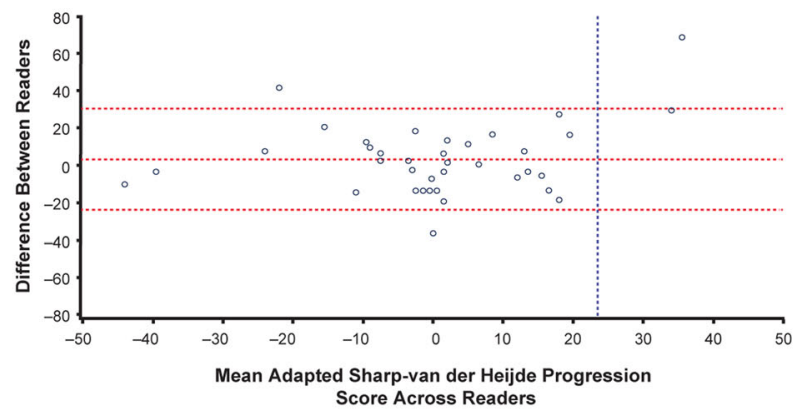

D

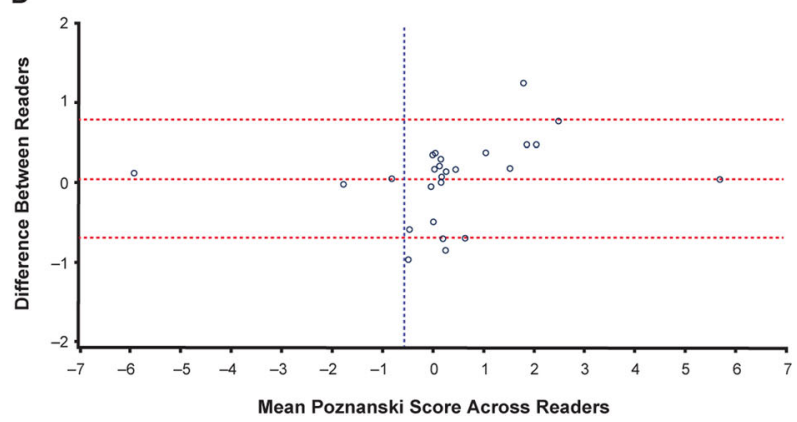

Fig. 1 Bland and Altman plots of radiographic progression based on SDD in patients with SJIA. a, b Mean adapted SH progression scores across two reviewers for each patient with radiographic scores at baseline and week $52(\mathbf{a}, n=45)$ and baseline and week $104(\mathbf{b}, n=37)$. $\mathbf{c}, \mathbf{d}$ Mean Poznanski score across two reviewers for each patient with Poznanski scores at baseline and week $52(\mathbf{c}, n=32)$ and baseline and week 104 (dd, $n=26)$. SDD thresholds are represented by vertical dashed lines. Patients with adapted SH progression are represented to the right of the vertical line in $\mathbf{a}$ and $\mathbf{b}$. Patients with Poznanski progression are represented to the left of the vertical line in $\mathbf{c}$ and $\mathbf{d}$. Horizontal dashed lines represent mean difference between readers \pm SDD. In case of discrepancy between readers, radiographs were adjudicated and reread independently so consensus could be reached. SDD, smallest detectable difference; SH, Sharp-van der Heijde; sJIA, systemic juvenile idiopathic arthritis 

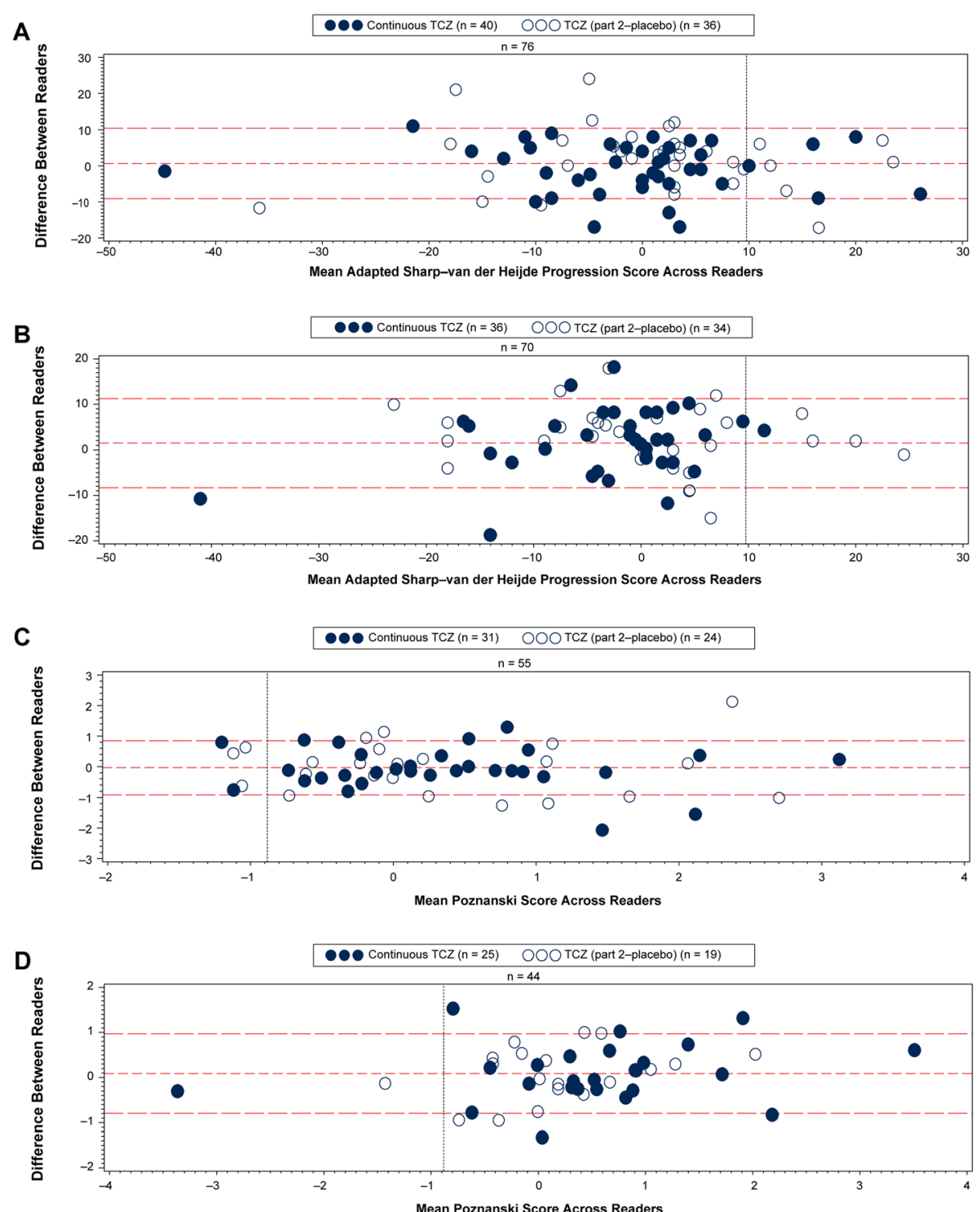

Fig. 2 Bland and Altman plots of radiographic progression based on SDD in patients with pcJIA. a, b Mean adapted SH progression scores across two reviewers for each patient with radiographic scores at baseline and week $52(\mathbf{a}, n=76)$ and baseline and week $104(\mathbf{b}, n=70)$. c, d Mean Poznanski scores across two reviewers for each patient with Poznanski scores at baseline and week $52(\mathbf{c}, n=55)$ and baseline and week 104 (d, $n=44)$. SDD thresholds are represented by vertical dashed lines. Patients with adapted SH progression are represented to the right of the vertical line in $\mathbf{a}$ and $\mathbf{b}$. Patients with Poznanski progression are represented to the left of the vertical line in $\mathbf{c}$ and $\mathbf{d}$. Patients randomly assigned to tocilizumab in part 2 are represented by filled circles; those randomly assigned to placebo in part 2 are represented by empty circles. Horizontal dashed lines represent mean differences between readers \pm SDD. In case of discrepancy between readers, radiographs were adjudicated and reread independently so consensus could be reached. PcJIA, polyarticular-course juvenile idiopathic arthritis; SDD, smallest detectable difference; SH, Sharp-van der Heijde; TCZ, tocilizumab

criteria. One of these patients also exhibited Poznanski progression. Of the other two patients with Poznanski progression at week 104, one achieved JIA ACR70 and the other achieved JIA ACR90 response.

\section{Radiographic progression in patients with pcliA}

There were nonsignificant changes in total, erosion, and JSN aSH scores from baseline to weeks 52 and 104
(Fig. 3c, Table 2). Patients receiving glucocorticoids at baseline had a positive median change in aSH score, whereas those not receiving glucocorticoids at baseline had a negative median change $(2.50$ vs -1.50$)$. No significant differences were observed for other parameters (data not shown). A significant positive change of 0.55 $(P=0.004)$ in Poznanski score was observed from baseline to week 104, and a positive, though not significant, 


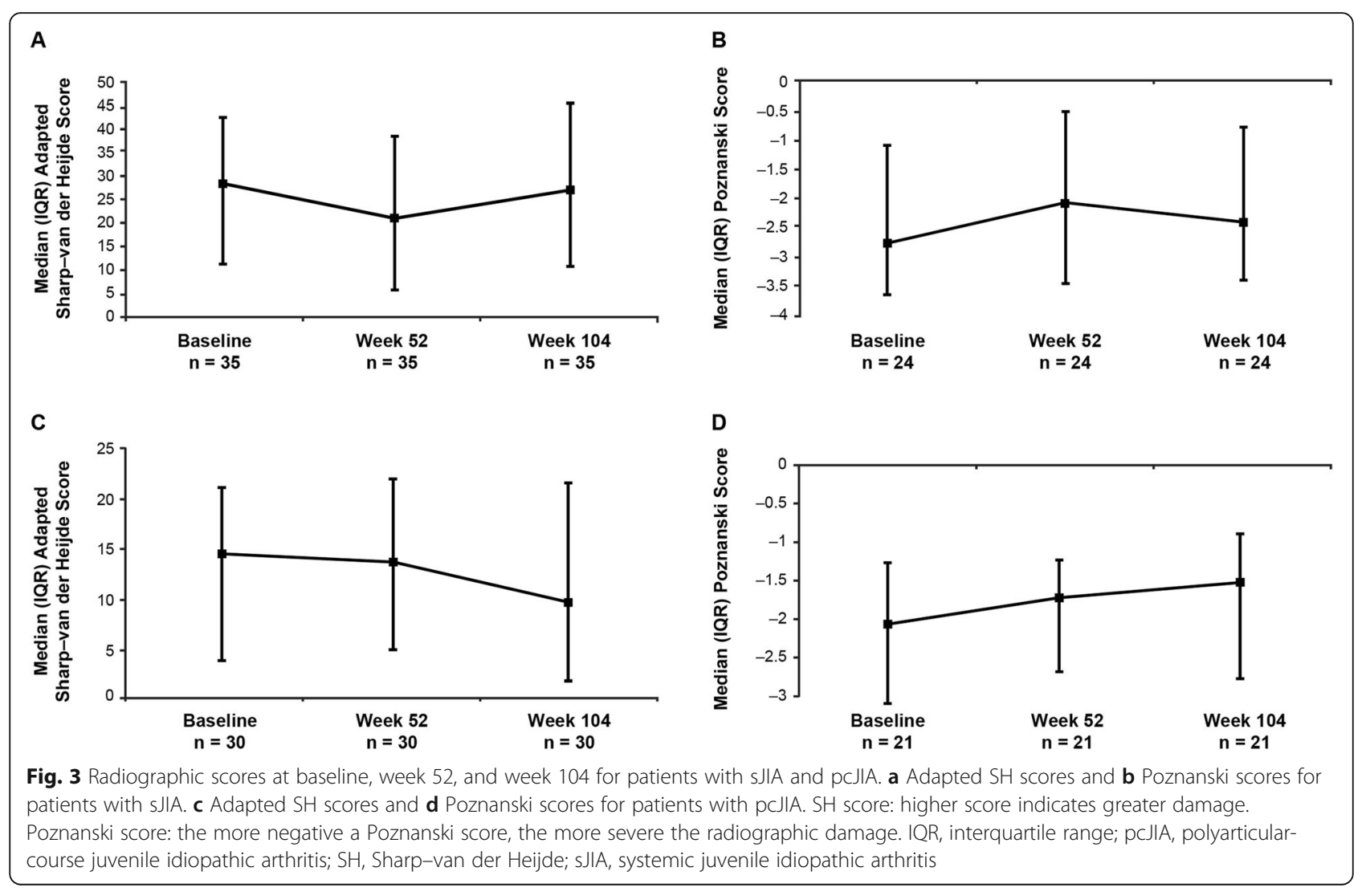

change of $0.26(P=0.077)$ was detected from baseline to week 52 (Fig. 3d, Table 2). There was a weak-tomoderate negative correlation between aSH and Poznanski scores at week 52 (Pearson correlation, 0.420; Spearman correlation, - 0.450) and week 104 (Pearson correlation, -0.522 ; Spearman correlation, $-0.429)$.
Based on week 104 data, SDD values were 9.76 and 0.88 for aSH and Poznanski progression, respectively. Using these SDDs, $87.5 \%$ and $97.1 \%$ of patients in the continuous TCZ population experienced no aSH progression at weeks 52 and 104, respectively, and one patient experienced progression after 2 years of tocilizumab treatment (Fig. 2a, b). Using the same SDD,

Table 2 Change from baseline in adapted SH and Poznanski scores

\begin{tabular}{|c|c|c|c|c|c|c|c|c|}
\hline \multirow[t]{2}{*}{ Score } & \multicolumn{2}{|c|}{ Baseline } & \multicolumn{3}{|c|}{ Change from baseline to week 52} & \multicolumn{3}{|c|}{ Change from baseline to week 104} \\
\hline & $n$ & Median (IQR) & $n$ & Median (IQR) & $P$ & $n$ & Median (IQR) & $P$ \\
\hline \multicolumn{9}{|l|}{ sJIA } \\
\hline Adapted total SH & 47 & $24.60(8.50,39.50)$ & 45 & $0.00(-8.70,4.00)$ & 0.302 & 37 & $0.50(-7.50,12.00)$ & 0.695 \\
\hline Erosion & 47 & $8.50(1.50,23.50)$ & 45 & $0.50(-3.50,1.50)$ & 0.677 & 37 & $0.50(-1.00,4.50)$ & 0.257 \\
\hline$J S N$ & 47 & $13.00(6.00,19.50)$ & 45 & $0.00(-4.00,3.00)$ & 0.257 & 37 & $0.00(-5.50,4.00)$ & 0.937 \\
\hline Poznanski & 33 & $-2.38(-3.48,-0.84)$ & 32 & $0.29(-0.05,1.05)$ & 0.003 & 26 & $0.16(-0.01,1.04)$ & 0.057 \\
\hline \multicolumn{9}{|l|}{ pcJIA } \\
\hline Adapted total SH & 45 & $8.00(3.00,18.50)$ & 40 & $0.50(-7.25,4.50)$ & 0.700 & 35 & $-1.00(-6.50,2.50)$ & 0.109 \\
\hline Erosion & 45 & $3.00(0.00,6.50)$ & 40 & $0.00(-1.50,1.50)$ & 0.819 & 36 & $0.00(-2.75,0.75)$ & 0.402 \\
\hline JSN & 45 & $5.00(1.00,14.50)$ & 40 & $0.25(-5.25,3.50)$ & 0.614 & 35 & $-1.00(-3.50,1.50)$ & 0.109 \\
\hline Poznanski & 35 & $-1.45(-2.51,-0.75)$ & 31 & $0.26(-0.34,0.91)$ & 0.077 & 25 & $0.55(0.04,0.92)$ & 0.004 \\
\hline
\end{tabular}

Abbreviations: IQR interquartile range, JSN joint space narrowing, $p c J I A$ polyarticular-course juvenile idiopathic arthritis, SH Sharp-van der Heijde, sJIA systemic juvenile idiopathic arthritis

SH score: higher score indicates greater damage. Poznanski score: the more negative a Poznanski score, the more severe the radiographic damage 
92.9\% of patients in the all TCZ population experienced no aSH progression at week 104 (Fig. 2b).

Using the SDD of $-0.88,96.0 \%$ of patients in the continuous TCZ population experienced no Poznanski progression at week 104 compared with $93.5 \%$ at week 52 (Fig. 2c, d). Applying the same SDD, $95.5 \%$ of patients in the all TCZ population experienced no Poznanski progression at week 104 (Fig. 2d).

Mean annualized progression rates from baseline to week 104 were calculated in the all TCZ population. Consistent with the continuous TCZ population, annualized progression rates for total, erosion, and JSN aSH scores were $-0.75,0.31$, and -0.95 , respectively, and the Poznanski score was 0.19 , indicating a lack of radiographic progression in the entire radiographic population over 2 years. The JIA ACR response rate was higher in patients without radiographic progression than in the entire study population at weeks 52 and 104 (Fig. 4a, b). The proportion of patients in each JIA ACR response category was maintained or increased from week 52 to week 104 and was greater at week 104 than the proportions in the continuous TCZ population, which included all patients randomly assigned to tocilizumab during part 2 , regardless of the availability of radiographic data (Fig. 4a, b).

\section{Discussion}

TENDER and CHERISH are the first trials in JIA to include radiologic analysis. Most patients with sJIA and
pcJIA treated with tocilizumab experienced no radiographic progression assessed by SDD and zero value methods. There was little change in radiographic scores of structural joint damage during 2 years of treatment. This is the first time that two separate phase 3 randomized controlled trials in patients with sJIA or pcJIA have been evaluated for X-ray progression within the framework of a pediatric investigation plan for the European Medicines Agency and a pediatric study plan for the US Food and Drug Administration.

The present analysis was not controlled; therefore, the possibility that patients had less aggressive illness than those who were not included in the analysis cannot be excluded. However, all JIA outcome measures at the start of treatment were comparable between patients included and excluded from the analysis. Furthermore, all patients had severe, long-standing systemic or polyarticular-course disease that was not controlled despite previous use of at least one traditional second-line agent, and all had bilateral wrist involvement. Patients with sJIA and pcJIA are more likely to develop destructive disease [30-32], and it has been suggested that patients with JIA and bilateral wrist disease are at high risk for radiographic progression [14, 33].

The observed changes in aSH score in pcJIA patients and the change in Poznanski score in sJIA and pcJIA patients suggest that at least some patients experienced

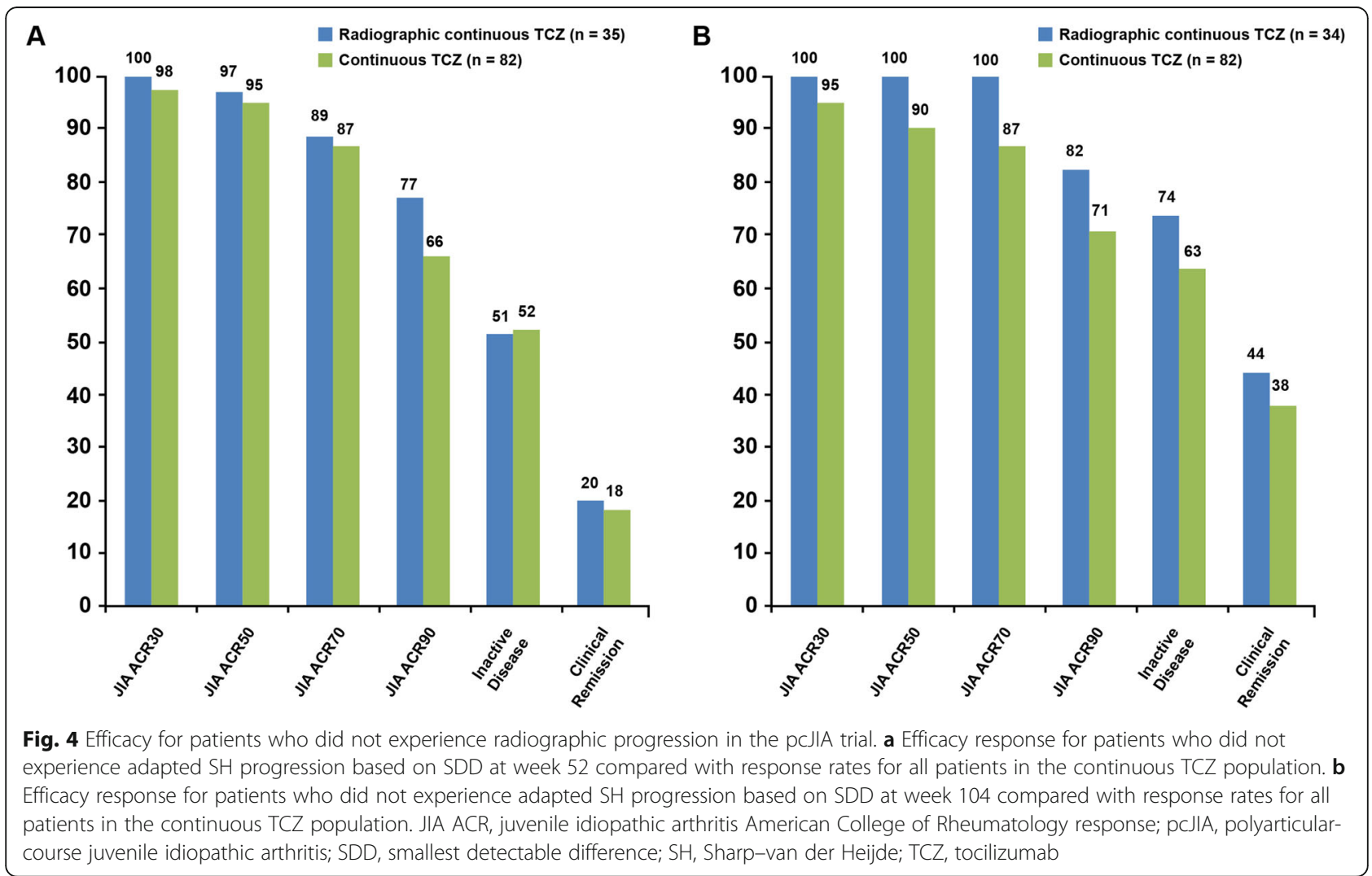


improvement of articular damage. Altogether, these findings indicate that tocilizumab is potentially capable of halting the progression of radiographic joint damage in children with JIA. Improvement in the rate of radiographic progression in children with JIA is not surprising because the regenerative capacity of articular cartilage is better in growing children than in adults [4].

These observations corroborate previous reports showing amelioration of radiographic joint changes with tocilizumab treatment in children with systemic JIA $[16,25]$. Coupled with previous demonstrations of the potential capacity of etanercept to repair radiographic joint damage in patients with JIA $[15,34]$, these observations indicate that biologic agents may have disease-modifying potential in JIA and underscore the need for randomized controlled trials to explore the capacity of biologics to prevent structural joint damage.

Given the high degree of concomitant methotrexate use among patients in the sJIA and the pcIIA studies, our data do not establish whether combination with methotrexate could enhance the effectiveness of tocilizumab. Literature is inconclusive on the effects of methotrexate on joint destruction, although two studies have suggested that this medication might have diseasemodifying potential $[11,13,14,35]$. Notably, all previous investigations of the effect of disease-modifying antirheumatic drugs on radiographic progression in JIA in wrist joints used the Poznanski score.

We used aSH and Poznanski scores to measure radiographic progression in the present analysis because these methods were specifically developed and validated for use in patients with JIA $[4,6,15]$. These measures significantly correlated with long-term joint damage and disability in physical function of children with pcJIA [6]. In the present analysis, radiography scores were reliable and showed good inter-reader and intra-reader agreement. In the absence of radiographic progression, approximately $50 \%$ of patients are expected to experience change from baseline of less than zero, and approximately $50 \%$ are expected to experience change greater than zero because of random errors introduced during radiographic reading. Therefore, the approximate 50:50 split between patients with and without radiographic progression at weeks 52 and 104 suggests there was little radiographic progression throughout the study, which is consistent with small changes in aSH score.

Limitations of the present analysis include the small number of patients in the radiographic populations of both trials. Limited availability of radiographs in the patient sample was due to nonparticipation of some study investigators in the radiographic study or lack of consent for radiographic assessments. Both scoring systems used to quantify radiographic damage evaluated wrist and hand joints, and it is unclear whether changes in these joints sufficiently reflect damage to large weight-bearing joints [4]. Some radiographs could not be assessed with the Poznanski method because of advanced bone erosions, which precluded a reliable definition of bone ends, or because of radiographic closure of growth plates of the second metacarpal bone in postpubertal patients. Lastly, we recognize that it was not possible to compare radiographic progression of tocilizumab-treated patients with that of patients who did not receive tocilizumab, particularly in the context of published data regarding the rate of radiographic progression in sJIA and pcJIA patients who did not receive tocilizumab.

\section{Conclusion}

In conclusion, this analysis of radiographic data from the TENDER and CHERISH trials suggests that tocilizumab may prevent radiographic progression in children with sJIA and children with pcJIA. However, because the natural course of arthritis in children is heterogeneous and a control group not exposed to tocilizumab was not available, we cannot draw definitive conclusions regarding the ability of tocilizumab to halt or diminish radiographic progression in JIA, and our findings should be confirmed in future studies.

\section{Supplementary information}

Supplementary information accompanies this paper at https://doi.org/10 1186/s13075-020-02303-y.

Additional file 1 Supplemental Methods. Inter-reader reliability methods for assessment of radiographs. Radiographic progression using cutoff of zero.

\section{Abbreviations}

ACR: American College of Rheumatology; aSH: Adapted Sharp-van der Heijde; CHAQ-DI: Childhood Health Assessment Questionnaire-Disability Index; DMARDs: Disease-modifying antirheumatic drugs; ESR: Erythrocyte sedimentation rate; GC: Glucocorticoid; ICC: Intra-class correlation coefficient; IL-6: Interleukin-6; IQR: Interquartile range; JIA ACR: Juvenile idiopathic arthritis American College of Rheumatology response; JSN: Joint space narrowing; LOM: Limitation of motion; M2: Second metacarpal bone; pcJIA: Polyarticular-course juvenile idiopathic arthritis; PRCSG: Pediatric Rheumatology Collaborative Study Group; PRINTO: Paediatric Rheumatology International Trials Organisation; RM: Radial metacarpal; SD: Standard deviation; SDD: Smallest detectable difference; SH: Sharp-van der Heijde; sJIA: Systemic juvenile idiopathic arthritis; TCZ: Tocilizumab; VAS: Visual analog scale

\section{Acknowledgements}

The authors thank Silvia Scala, Irene Gregorini, Laura Carenini, Mariangela Rinaldi, and Elisa Patrone of the PRINTO coordinating center in Genoa, Italy, for overall coordination of the project and Caroline Keane of Roche Products Ltd for assistance with data analysis.

Medical writing support was provided by Sara Duggan, PhD, of ApotheCom and was funded by Roche.

\section{Authors' contributions}

The study was designed jointly by academic authors (AR, NR, CM) and F. Hoffmann-La Roche Ltd (Roche), with data collected by PRINTO and PRCSG investigators. X-ray readings were performed by $C M$ and $A R$ with assistance for statistical analysis provided by AP and NR. Roche provided the funding, overall management, and data analysis. The first and subsequent versions of 
the manuscript were written by $A R, N R$, and $C M$; edited by HIB and FDB; and revised critically by all remaining authors and Roche. All academic authors vouch for the completeness and veracity of the data and data analyses. All authors reviewed and approved the final draft of the manuscript for submission for publication.

\section{Authors' information}

Not applicable

\section{Funding}

This study was funded by F. Hoffmann-La Roche Ltd. Support for third-party medical writing assistance was provided by F. Hoffmann-La Roche Ltd. The sponsor was involved in the study design, analysis and interpretation of data, manuscript writing, and final decision to submit the manuscript for publication.

\section{Availability of data and materials}

Qualified researchers may request access to data through the clinical study data request platform (www.clinicalstudydatarequest.com). Further details on Roche's criteria for eligible studies are available here: https://

clinicalstudydatarequest.com/Study-Sponsors/Study-Sponsors-Roche.aspx. For further details on Roche's Global Policy on the Sharing of Clinical Information and how to request access to related clinical study documents, see here: https://www.roche.com/research_and_development/who_we_are_how_we_ work/clinical_trials/our_commitment_to_data_sharing.htm.

\section{Ethics approval and consent to participate}

Both trials included in this manuscript were conducted in accordance with the Declaration of Helsinki and good clinical practice and were approved by local institutional review boards or independent ethics committees at each center. Parents or guardians provided written informed consent, and patients provided written informed assent according to national requirements.

\section{Consent for publication}

Not applicable

\section{Competing interests}

CM has received consulting fees $(<\$ 10,000)$ from AbbVie

NR has received honoraria for consultancies or speaker bureaus $(<\$ 10,000)$ from the following in the past 2 years: Ablynx, AbbVie, Boehringer, BristolMyers Squibb, Eli Lilly, EMD Serono, F. Hoffmann-La Roche, Janssen, Merck, Novartis, Pfizer, Rewind, R-Pharma, Sanofi, Servier, Sinergie, and Takeda. The Gaslini Hospital, where NR works as a full-time public employee, has received contributions $(>\$ 10,000)$ from the following industries in the last 2 years: $A b-$ bott, Bristol-Myers Squibb, The Angelini Group "Francesco Angelini," GlaxoSmithKline, F. Hoffmann-La Roche, Italfarmaco, Janssen, Novartis, Pfizer, Sanofi Aventis, Schwarz Biosciences, Sobi, Xoma, and Wyeth. This funding has been reinvested for the research activities of the hospital in a fully independent manner without any commitment to third parties.

$S P, E P, A P, B F, S G$, and GG declare no competing interests.

CWouters declares unrestricted research grants to her institution from GlaxoSmithKline, Pfizer, and Roche.

PD has received speaker or consultant fees from AbbVie, Novartis, Pfizer, F. Hoffmann-La Roche, Medac, Eli Lilly, Sanofi, and Merck Sharp \& Dohme $(<\$ 10,000)$.

CWells is an employee of Roche Products Ltd.

WD is an employee of and owns stock in Roche Products Ltd. HIB has received consulting fees $(<\$ 10,000)$ from Pfizer, EMD Serono, and AstraZeneca and $(>\$ 10,000)$ from Bristol-Myers Squibb and Janssen and research grants from Bristol-Myers Squibb and Pfizer.

FDB has received consulting fees $(<\$ 10,000)$ from F. Hoffmann-La Roche and an unrestricted research grant from F. Hoffmann-La Roche.

AR has received consulting fees $(<\$ 10,000)$ from AbbVie, Bristol-Myers Squibb, Pfizer, F. Hoffmann-La Roche, Novartis, Centocor, Francesco Angelini, and Reckitt Benckiser.

\section{Author details}

${ }^{1}$ Università degli Studi di Genova, Genoa, Italy. ${ }^{2}$ IRCCS Istituto Giannina Gaslini, Clinica Pediatrica e Reumatologia, PRINTO, Via G. Gaslini 5, 16147 Genoa, Italy. ${ }^{3}$ IRCCS Istituto Giannina Gaslini, Servizio di Epidemiologia e Biostatistica, Genoa, Italy. ${ }^{4}$ University Hospital Gasthuisberg, Leuven, Belgium. ${ }^{5}$ General University Hospital and First Faculty of Medicine, Charles University,
Prague, Czech Republic. ${ }^{6}$ Oslo University Hospital and University of Oslo, Oslo, Norway. ${ }^{7}$ Hospital Sor Maria Ludovica, La Plata, Argentina. ${ }^{8}$ Roche Products Ltd., Welwyn Garden City, UK. ${ }^{9}$ Cincinnati Children's Hospital Medical Center, Cincinnati, OH, USA. ${ }^{10}$ IRCCS Ospedale Pediatrico Bambino Gesú, Rome, Italy. ${ }^{11}$ Sechenov First Moscow State Medical University, Moscow, Russian Federation.

\section{Received: 18 October 2019 Accepted: 24 August 2020 \\ Published online: 10 September 2020}

\section{References}

1. Petty RE, Southwood TR, Manners P, Baum J, Glass DN, Goldenberg J, et al. International League of Associations for Rheumatology classification of juvenile idiopathic arthritis: second revision, Edmonton, 2001. J Rheumatol. 2004:31(2):390-2.

2. Ravelli A, Martini A. Juvenile idiopathic arthritis. Lancet. 2007;369(9563):767-78.

3. Oen K, Reed M, Malleson PN, Cabral DA, Petty RE, Rosenberg AM, et al. Radiologic outcome and its relationship to functional disability in juvenile rheumatoid arthritis. J Rheumatol. 2003;30(4):832-40.

4. Ravelli A. The time has come to include assessment of radiographic progression in juvenile idiopathic arthritis clinical trials. J Rheumatol. 2008; 35(4):553-7.

5. van Rossum MA, Zwinderman AH, Boers M, Dijkmans BA, van Soesbergen RM, Fiselier TJ, et al. Radiologic features in juvenile idiopathic arthritis: a first step in the development of a standardized assessment method. Arthritis Rheum. 2003:48(2):507-15.

6. Magni-Manzoni S, Rossi F, Pistorio A, Temporini F, Viola S, Beluffi G, et al. Prognostic factors for radiographic progression, radiographic damage, and disability in juvenile idiopathic arthritis. Arthritis Rheum. 2003;48(12):3509-17.

7. Doria AS, de Castro CC, Kiss MH, Sernik RA, Vitule LF, Silva CH, et al. Interand intrareader variability in the interpretation of two radiographic classification systems for juvenile rheumatoid arthritis. Pediatr Radiol. 2003; 33(10):673-81.

8. Mason T, Reed AM, Nelson AM, Thomas KB. Radiographic progression in children with polyarticular juvenile rheumatoid arthritis: a pilot study. Ann Rheum Dis. 2005;64(3):491-3.

9. van Rossum MA, Boers $M$, Zwinderman $A H$, van Soesbergen RM, Wieringa $\mathrm{H}$, Fiselier TJ, et al. Development of a standardized method of assessment of radiographs and radiographic change in juvenile idiopathic arthritis: introduction of the Dijkstra composite score. Arthritis Rheum. 2005;52(9): 2865-72.

10. Rossi F, Di Dia F, Galipò O, Pistorio A, Valle M, Magni-Manzoni S, et al. Use of the Sharp and Larsen scoring methods in the assessment of radiographic progression in juvenile idiopathic arthritis. Arthritis Rheum. 2006;55(5):717-23.

11. Ravelli A, loseliani M, Norambuena X, Sato J, Pistorio A, Rossi F, et al. Adapted versions of the Sharp/van der Heijde score are reliable and valid for assessment of radiographic progression in juvenile idiopathic arthritis. Arthritis Rheum. 2007;56(9):3087-95.

12. Rodriguez-Lozano AL, Giancane G, Pignataro R, Viola S, Valle M, Gregorio S, et al. Agreement among musculoskeletal pediatric specialists in the assessment of radiographic joint damage in juvenile idiopathic arthritis. Arthritis Care Res. 2014;66(1):34-9.

13. Harel L, Wagner-Weiner L, Poznanski AK, Spencer CH, Ekwo E, Magilavy DB. Effects of methotrexate on radiologic progression in juvenile rheumatoid arthritis. Arthritis Rheum. 1993;36(10):1370-4.

14. Ravelli A, Viola S, Ramenghi B, Beluffi G, Zonta LA, Martini A. Radiologic progression in patients with juvenile chronic arthritis treated with methotrexate. J Pediatr. 1998;133(2):262-5.

15. Nielsen S, Ruperto N, Gerloni V, Simonini G, Cortis E, Lepore L, et al. Preliminary evidence that etanercept may reduce radiographic progression in juvenile idiopathic arthritis. Clin Exp Rheumatol. 2008;26(4):688-92.

16. Aoki C, Inaba Y, Choe H, Kaneko U, Hara R, Miyamae T, et al. Discrepancy between clinical and radiological responses to tocilizumab treatment in patients with systemic-onset juvenile idiopathic arthritis. J Rheumatol. 2014; 41(6):1171-7.

17. Nishimoto N, Kishimoto T. Humanized antihuman IL-6 receptor antibody, tocilizumab. Handb Exp Pharmacol. 2008;181:151-60.

18. Mihara M, Kasutani K, Okazaki M, Nakamura A, Kawai S, Sugimoto M, et al. Tocilizumab inhibits signal transduction mediated by both mIL-6R and sIL$6 \mathrm{R}$, but not by the receptors of other members of IL-6 cytokine family. Int Immunopharmacol. 2005;5(12):1731-40. 
19. De Benedetti F, Brunner HI, Ruperto N, Kenwright A, Wright S, Calvo I, et al. Randomized trial of tocilizumab in systemic juvenile idiopathic arthritis. N Engl J Med. 2012;367(25):2385-95.

20. Brunner HI, Ruperto N, Zuber Z, Keane C, Harari O, Kenwright A, et al. Efficacy and safety of tocilizumab in patients with polyarticular-course juvenile idiopathic arthritis: results from a phase 3, randomised, doubleblind withdrawal trial. Ann Rheum Dis. 2014;74(6):1110-7.

21. Actemra (tocilizumab) injection for intravenous or subcutaneous use [prescribing information]. South San Francisco: Genentech Inc.; 2018.

22. Fleischmann RM, Halland AM, Brzosko M, Burgos-Vargas R, Mela C, Vernon E, et al. Tocilizumab inhibits structural joint damage and improves physical function in patients with rheumatoid arthritis and inadequate responses to methotrexate: LITHE study 2-year results. J Rheumatol. 2013;40(2):113-26.

23. Kremer JM, Blanco R, Brzosko S, Burgos-Vargas R, Halland AM, Vernon E, et al. Tocilizumab inhibits structural joint damage in rheumatoid arthritis patients with inadequate responses to methotrexate: results from the double-blind treatment phase of a randomized placebo-controlled trial of tocilizumab safety and prevention of structural joint damage at one year. Arthritis Rheum. 2011;63(3):609-21.

24. Nishimoto N, Hashimoto J, Miyasaka N, Yamamoto K, Kawai S, Takeuchi T, et al. Study of active controlled monotherapy used for rheumatoid arthritis, an IL-6 inhibitor (SAMURAI): evidence of clinical and radiographic benefit from an $x$-ray reader-blinded randomised controlled trial of tocilizumab. Ann Rheum Dis. 2007:66(9):1162-7.

25. Inaba Y, Ozawa R, Imagawa T, Mori M, Hara Y, Miyamae T, et al. Radiographic improvement of damaged large joints in children with systemic juvenile idiopathic arthritis following tocilizumab treatment. Ann Rheum Dis. 2011;70(9):1693-5.

26. Ruperto N, Martini A. Networking in paediatrics: the example of the Paediatric Rheumatology International Trials Organisation (PRINTO). Arch Dis Child. 2011;96(6):596-601.

27. Poznanski AK, Hernandez RJ, Guire KE, Bereza UL, Garn SM. Carpal length in children - a useful measurement in the diagnosis of rheumatoid arthritis and some congenital malformation syndromes. Radiology. 1978;129(3):661-8.

28. Lassere M, Boers M, van der Heijde D, Boonen A, Edmonds J, Saudan A, et al. Smallest detectable difference in radiological progression. J Rheumatol. 1999;26(3):731-9.

29. Giannini EH, Ruperto N, Ravelli A, Lovell DJ, Felson DT, Martini A. Preliminary definition of improvement in juvenile arthritis. Arthritis Rheum. 1997;40(7): 1202-9.

30. Oen K. Long-term outcomes and predictors of outcomes for patients with juvenile idiopathic arthritis. Best Pract Res Clin Rheumatol. 2002;16(3):347-60.

31. Ravelli A. Toward an understanding of the long-term outcome of juvenile idiopathic arthritis. Clin Exp Rheumatol. 2004;22(3):271-5.

32. Levinson JE, Wallace CA. Dismantling the pyramid. J Rheumatol Suppl. 1992; 33:6-10.

33. Cassone R, Falcone A, Rossi F, Magni-Manzoni S, Felici E, Buoncompagni A, et al. Unilateral destructive wrist synovitis in juvenile idiopathic arthritis. Clin Exp Rheumatol. 2004;22(5):637-42.

34. Remy A, Combe B. Repair of radiographic hip joint in juvenile rheumatoid arthritis patients treated with etanercept plus methotrexate. Joint Bone Spine. 2014:81(5):447-9.

35. Giancane G, Pederzoli S, Norambuena X, loseliani M, Sato J, Gallo MC, et al, Frequency of radiographic damage and progression in individual joints in children with juvenile idiopathic arthritis. Arthritis Care Res. 2014;66(1):27-33.

\section{Publisher's Note}

Springer Nature remains neutral with regard to jurisdictional claims in published maps and institutional affiliations.

Ready to submit your research? Choose BMC and benefit from:
- fast, convenient online submission
- thorough peer review by experienced researchers in your field
- rapid publication on acceptance
- support for research data, including large and complex data types
- gold Open Access which fosters wider collaboration and increased citations
- maximum visibility for your research: over 100M website views per year
At BMC, research is always in progress.
Learn more biomedcentral.com/submissions

